\title{
Homeostatic Regulation of Sleep: A Role for Preoptic Area Neurons
}

\author{
Irma Gvilia, ${ }^{1,2,4}$ Feng Xu, ${ }^{1}$ Dennis McGinty, ${ }^{1,3}$ and Ronald Szymusiak ${ }^{1,2}$ \\ ${ }^{1}$ Research Service, Veterans Affairs Greater Los Angeles Healthcare System, North Hills, California 91343, Departments of ${ }^{2}$ Medicine and ${ }^{3}$ Psychology, \\ University of California, Los Angeles, California 90095, and ${ }^{4}$ I. Beritashvili Institute of Physiology, Tbilisi 0160, Georgia
}

The median preoptic nucleus (MnPN) and the ventrolateral preoptic area (vlPOA) contain putative sleep-regulatory neurons that exhibit elevated discharge rates during sleep compared with waking. Expression of c-Fos protein immunoreactivity (IR) in GABAergic neurons in the MnPN and the vlPOA is high in spontaneously sleeping rats and in rats undergoing recovery sleep after sleep deprivation. However, it is unclear whether c-Fos-IR in these neurons is evoked by increases in sleep pressure or by increases in sleep amount. We examined c-Fos-IR in MnPN and vlPOA neurons under experimental conditions that dissociated homeostatic sleep pressure, sleep amount, and time of day. Groups of rats with strong diurnal rhythms in sleep-wake organization were killed after (1) spontaneous sleep in the light, (2) spontaneous sleep in the dark, (3) sleep deprivation (SLD) in the light and (4) recovery sleep after SLD in the light. Numbers of GABAergic neurons expressing c-Fos-IR in the MnPN were significantly higher after SLD in the light compared with spontaneous sleep and recovery sleep in the light. In contrast, Fos-IR in vlPOA GABAergic neurons was most prevalent after spontaneous sleep and recovery sleep in the light. No light-dark differences in Fos-IR were observed in the MnPN after SLD in groups of rats with weak or absent diurnal sleep-waking rhythms. Our findings define potential roles for MnPN and vlPOA GABAergic neurons in homeostatic aspects of sleep regulation.

Key words: sleep deprivation; non-REM sleep; ventrolateral preoptic area; median preoptic nucleus; GABA; sleep diurnality

\section{Introduction}

The median preoptic nucleus $(\mathrm{MnPN})$ and the ventral lateral preoptic area (vlPOA) of the hypothalamus contain sleep-active neurons. Both the MnPN and the vlPOA exhibit sleep-associated c-Fos protein immunoreactivity (Fos-IR) (Sherin et al., 1996, 1998; Gong et al., 2000) and subsets of neurons in these nuclei have elevated discharge rates during sleep compared with waking as demonstrated by unit-recording studies (Szymusiak et al., 1998; Suntsova et al., 2002). Sleep-related Fos-IR is predominantly expressed in MnPN GABAergic cells and in GABAergic/ galaninergic vlPOA neurons (Sherin et al., 1998; Gaus et al., 2002; Gong et al., 2004). The number of Fos-positive GABAergic cells in these nuclei is positively correlated with the total amount of preceding sleep (Gong et al., 2004).

Original studies on functional mapping of Fos-IR reported low levels of Fos-positive neurons in the MnPN of rats that were sleep deprived compared with spontaneously sleeping rats (Gong et al., 2000), whereas recent studies have reported higher numbers of Fos-immunoreactive neurons (IRNs) in the MnPN of sleep-deprived rats (Modirrousta et al., 2004; Peterfi et al., 2004;

Received May 10, 2006; revised July 20, 2006; accepted Aug. 7, 2006.

This work was supported by the Medical Research Service of the Department of Veterans Affairs and National Institutes of Health Grants MH63323 and HL60296. We thank Keng-Tee Chew and Bryan Angara for technical assistance.

Correspondence should be addressed to either Ronald Szymusiak or Irma Gvilia, Research Service (151A3), Veterans Affairs Greater Los Angeles Healthcare System, 16111 Plummer Street, North Hills, CA 19343. E-mail: rszym@ucla.edu or irmagvilia@hotmail.com.

DOI:10.1523/JNEUROSCI.2012-06.2006

Copyright $\odot 2006$ Society for Neuroscience $\quad$ 0270-6474/06/269426-08\$15.00/0
Gvilia et al., 2005) and of rapid eye movement (REM) sleepdeprived rats (Gvilia et al., 2006). The number of MnPN and vlPOA GABAergic neurons manifesting Fos-IR is elevated during recovery sleep after total sleep deprivation (Gong et al., 2004) and during recovery sleep after REM-sleep restriction (Gvilia et al., 2006). These findings indicate that elevated homeostatic pressure for non-REM and REM sleep can be associated with increased activation of subsets of MnPN and vlPOA neurons, suggesting an involvement of these neurons in the homeostatic regulation of sleep.

Little is known about specific neuronal systems that regulate homeostatic aspects of sleep control. The goal of the present study was to evaluate a hypothesis that activation of GABAergic neurons in the MnPN and vlPOA is associated with increasing homeostatic sleep drive/pressure and not with increasing sleep amount. Patterns of c-Fos-IR were compared among groups of rats exhibiting different levels of sleep pressure and different amounts of sleep. Experiment 1 used groups of rats with inherently strong diurnal rhythms in sleep-waking organization, with the assumption that such rats have comparatively high homeostatic sleep pressure during the light/rest period compared with the dark/active phase. Experiment 2 used rats with inherently weak diurnal rhythms in the distribution of sleep and waking, with the assumption that homeostatic sleep pressure in such rats is similar during the light and dark periods. Results indicate that MnPN GABAergic neurons are most strongly activated in response to increasing sleep pressure, whereas vlPOA GABAergic neurons are most strongly activated in response to increasing sleep amount. 
Table 1. Percentage of time spent in wakefulness, REM sleep, and non-REM sleep and mean numbers of non-REM sleep onsets

\begin{tabular}{|c|c|c|c|c|}
\hline & $\begin{array}{l}\text { Spontaneous sleep-waking during } \\
\text { the dark period }(n=6)\end{array}$ & $\begin{array}{l}\text { Spontaneous sleep-waking during } \\
\text { the light period }(n=6)\end{array}$ & $\begin{array}{l}\text { Sleep deprivation in the } \\
\text { light period }(n=6) \text {, } \\
\text { experiment } 1\end{array}$ & $\begin{array}{l}\text { Recovery after sleep deprivation } \\
(n=6) \text {, experiment } 1\end{array}$ \\
\hline Wakefulness (\%) & $91.2 \pm 3.4^{* *}$ & $27.1 \pm 2.6^{*}$ & $94.4 \pm 1.4^{* *}$ & $11.24 \pm 1.36^{*}$ \\
\hline REM sleep (\%) & $2 \pm 0.71^{*}$ & $16 \pm 1.67$ & 0 & $16.78 \pm 2.39$ \\
\hline Non-REM sleep (\%) & $6.8 \pm 2.9^{* *}$ & $57.7 \pm 2.04^{*}$ & $5.6 \pm 1.4^{* *}$ & $72.1 \pm 2.5^{*}$ \\
\hline \multicolumn{5}{|c|}{ Mean numbers of non-REM sleep } \\
\hline onsets (n) & $4 \pm 0.4^{*}$ & $9 \pm 0.5^{*}$ & $54 \pm 4.6^{*}$ & $14 \pm 0.5^{*}$ \\
\hline
\end{tabular}

Shown is the percentage of time spent in wakefulness, REM sleep, and non-REM sleep and mean numbers of non-REM sleep onsets for rats that were allowed spontaneous sleep-waking behavior during either the dark period ( $n=6$ ) or during the light period $(n=6)$, rats subjected to sleep deprivation during the light period $(n=6)$, and rats that were allowed recovery sleep after the sleep deprivation in the light $(n=6)$. Percentage of sleep-waking stages and the numbers of non-REM sleep onsets were calculated for a $1 \mathrm{~h}$ recording period before the rats were killed. ANOVA indicated significant effect of experimental condition for percentage of wakefulness $\left(F_{(3,20)}=356.1 ; p<0.001\right)$, percentage of non-REM sleep $\left(F_{(3,20)}=238.7 ; p<0.001\right)$ and percentage of REM sleep $\left(F_{(2,15)}=22.9 ; p<0.001\right)$. ANOVA also indicated significant effect of experimental conditions on the numbers of non-REM sleep onsets $\left(F_{(3,20)}=21.7 ; p<0.001\right)$. ${ }^{*}$ Significantly different from all other groups; ${ }^{* *}$ significantly different from the light-sleep and recovery sleep rats, $p<0.050$ (Newman-Keuls test).

Table 2. Mean numbers of attempts to enter into sleep during sleep deprivation periods and mean numbers of single Fos-IR and Fos plus GAD double IR neurons

\begin{tabular}{|c|c|c|c|c|c|}
\hline Experimental groups & No. of sleep attempts & No. of Fos IRNs & & No. of Fos plus $G$ & \\
\hline & & $\mathrm{rMnPN}$ & $\mathrm{CMnPN}$ & $\mathrm{rMnPN}$ & $\mathrm{CMnPN}$ \\
\hline Light SLD, $\exp 1(n=6)$ & $81 \pm 5.4^{*}$ & $87.83 \pm 3.8^{*}$ & $74.29 \pm 6.1^{*}$ & $42.19 \pm 2.8^{*}$ & $34.92 \pm 2.8^{*}$ \\
\hline Light SLD, $\exp 2(n=5)$ & $45.1 \pm 5.2$ & $58.64 \pm 6.4$ & $46.84 \pm 7.8$ & $13.16 \pm 2.4$ & $10 \pm 2.3$ \\
\hline Dark SLD, $\exp 2(n=4)$ & $36.75 \pm 7.2$ & $61 \pm 9.2$ & $51.49 \pm 5.7$ & $10.75 \pm 1.8$ & $11.73 \pm 2.3$ \\
\hline
\end{tabular}

Shown is the mean numbers of attempts to enter into sleep during sleep deprivation periods and mean numbers of single Fos-IR and Fos plus GAD double IR neurons in the MnPN for rats that were light-sleep deprived in the condition of a strong diurnal rhythm in baseline sleep-waking organization (experiment 1) and rats that were subjected to sleep deprivation either during the light period or during the dark period in the condition of weaker diurnal rhythms in baseline sleep and waking (experiment 2). Mean numbers of sleep attempts/entries were calculated for a $2 \mathrm{~h}$ recording period before the rats were killed. ANOVA indicated significant effect of experimental condition on the number of sleep attempts $\left(F_{(2,12)}=16.951 ; p<0.001\right)$. For single Fos-IR cell counts, ANOVA indicated significant effects of experimental conditions in both the rostral $\left(F_{(2,12)}=7.47 ; p<0.01\right)$ and caudal MnPN $\left(F_{(2,12)}=5.17 ; p<0.05\right)$. Similarly, there were significant effects of experimental conditions for Fos plus GAD IR cell counts in both rostral $\left(F_{(2,12)}=50.12 ; p<0.001\right)$ and caudal MnPN $\left(F_{(2,12)}=31.83 ; p<0.001\right)$. ${ }^{*}$ Significantly different from all other groups, $p<0.05$ (Newman-Keuls). exp, Experiment; No., number.

\section{Materials and Methods}

All experiments were approved by the Animal Care and Use Committee at the Veterans Affairs Greater Los Angeles Health Care System and were conducted according to the guidelines of the National Research Council.

\section{Animals and experimental environment}

Thirty-three male Sprague Dawley rats, weighing 280-320 g at the beginning of the experiments, were acclimated to a $12 \mathrm{~h}$ light/dark cycle (lights on at 8:00 A.M.). The rats were housed individually in environmental chambers. Food and water were available ad libitum and the ambient temperature was maintained at $23 \pm 0.5^{\circ} \mathrm{C}$.

\section{Surgical procedures and recording}

Under ketamine/xylazine anesthesia (80/10 mg/kg, i.p.), rats were surgically implanted with chronic cortical electroencephalogram (EEG) and dorsal neck electromyogram (EMG) electrodes for assessment of sleepwakefulness states. Briefly, stainless steel screw electrodes were implanted in the skull for EEG recordings and flexible insulated stainless steel wires were threaded into neck muscles for EMG recordings. Leads from the electrodes were soldered to a small Amphenol connector and the complete assembly was anchored to the skull with dental acrylic.

On the 8th-13th days after surgery, animals were connected to a recording cable that was lightly suspended above them by a counterweighted beam and were adapted to the recording procedure for $5-6 \mathrm{~h}$ (beginning at 8:00 A.M.) each day. During experiments, EEG and EMG signals were recorded continuously using a polysomnographic recording device (Embla; Medcare Flaga Medical Devices, Reykjavik, Iceland). The EEG and EMG signals were digitally displayed and stored on a computer using Somnologica software (Somnologica Studio; Medcare Flaga Medical Devices).

\section{Experimental paradigm}

On the 14th day after surgery, all rats $(n=33)$ were recorded for $24 \mathrm{~h}$ to determine baseline diurnal organization of sleep and waking. Rats $(n=$ 24) exhibiting $\geq 60 \%$ sleep during the light period and $\leq 30 \%$ sleep in the dark were assigned for experiment 1 , whereas rats $(n=9)$ with weaker diurnal rhythms in sleep and waking were assigned for experiment 2 . All other experimental recordings described below were conducted on the 15th day after surgery.

Experiment 1. One group of rats $(n=6)$ was allowed $2 \mathrm{~h}$ spontaneous
A: rats $(n=6)$ with strong diurnal rhythmsin sleep and waking

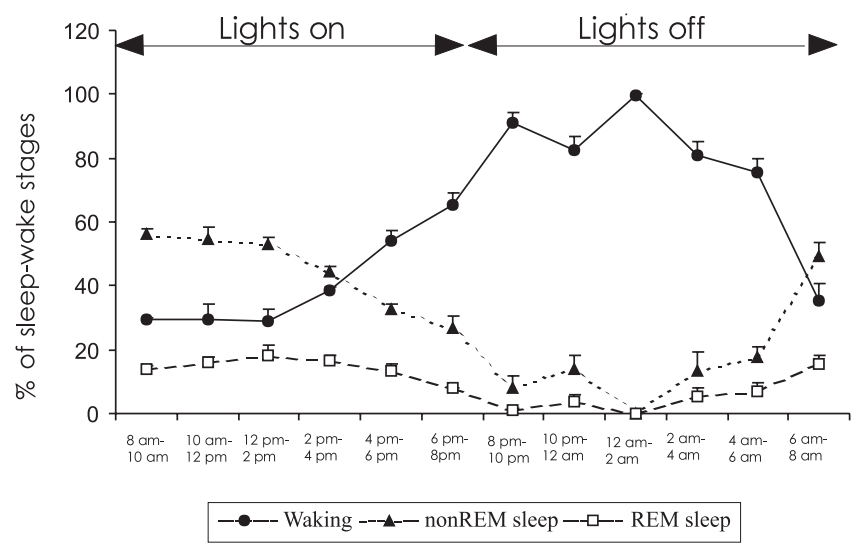

B: rats $(n=6)$ with weak diurnal rhythmsin sleep and waking

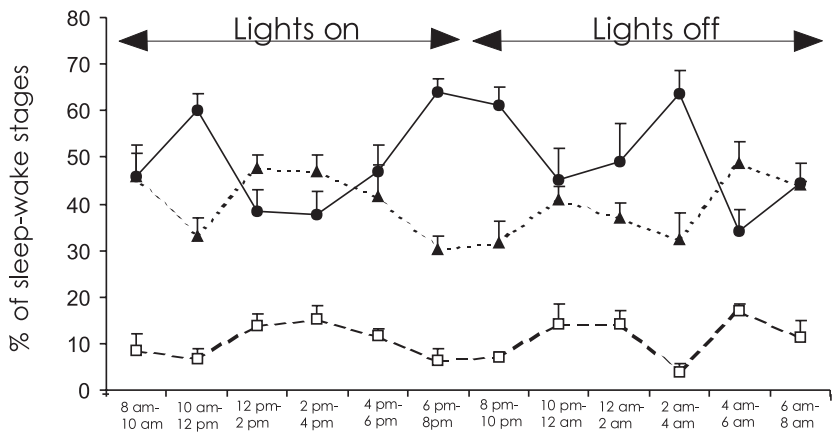

Figure 1. Mean percentages of time spent in waking, non-REM sleep, and REM sleep during $24 \mathrm{~h}$ baseline recordings for six rats exhibiting strong diurnal organization of sleep and wakefulness that were assigned to experiment $1(\boldsymbol{A})$ and six rats exhibiting weak diurnal organization of sleep and wakefulness that were assigned to experiment 2 (B). 
sleep and waking during the light period (ZT1$3)$. A second group of rats $(n=6)$ was allowed $2 \mathrm{~h}$ spontaneous sleep-waking behavior during the dark period (ZT13-15). A third group of rats $(n=12)$ was subjected to $2 \mathrm{~h}$ sleep deprivation during the light period (ZT1-3) and then divided into two subgroups: rats $(n=6)$ that were killed right after the termination of the deprivation procedure and rats $(n=6)$ that were allowed $1 \mathrm{~h}$ recovery sleep after the deprivation. To interrupt sleep episodes, rats were subjected to gentle arousing stimuli (tapping on the cage and/or slight movement of the cage) within 3-5 s of the first appearance of EEG signs of sleep. Animals were adapted to the sleep deprivation procedures on the 5th-13th postsurgical days, by exposing them to the stimuli used to interrupt sleep for several 5-10 min periods each day, between 8:00 A.M. and 3:00 P.M.

Experiment 2. A group of nine rats, exhibiting a weak diurnal rhythm in sleep-waking organization, was subjected to $2 \mathrm{~h}$ sleep deprivation during either the light period (ZT1-3; $n=5)$ or in the dark period (ZT13-15; $n=4)$. Immediately after the end of all recordings, rats were given a lethal dose of anesthetic followed by perfusion.

\section{Immunohistochemistry}

Under deep pentobarbital anesthesia $(100 \mathrm{mg} /$ $\mathrm{kg}$ ), animals were transcardially perfused with $0.12 \mathrm{M}$ Millonig's phosphate buffer (MPB) for 5 min, followed by $500 \mathrm{ml}$ of $4 \%$ paraformaldehyde (PFA) in $0.12 \mathrm{M}$ MPB. After perfusion, the bodies of the perfused animals were kept at $4^{\circ} \mathrm{C}$ for $1 \mathrm{~h}$. The brains were then removed, postfixed in the same PFA solution for $1 \mathrm{~h}$, washed in $0.12 \mathrm{M} \mathrm{MPB}$ and transferred successively to 10,20 , and $30 \%$ sucrose at $4^{\circ} \mathrm{C}$ until they sank. Brain tissue was processed for double immunostaining for Fos, the protein product of the immediate-early gene c-fos (Morgan and Curran, 1986; Dragunow and Faull, 1989), and for glutamic acid decarboxylase (GAD), a marker of GABAergic cells (see Fig. $4 D$ ). Thirty micrometers coronal sections were cut through the MnPN and the vlPOA on a freezing microtome. The sections were processed for Fos-protein staining first. Sections were incubated overnight in a rabbit anti-c-Fos primary antiserum (AB-5, 1:15,000; Oncogene Science, Cambridge, MA) on a shaking table, at $4^{\circ} \mathrm{C}$. Sections were processed with biotinylated goat anti-rabbit IgG (1:800; Vector Laboratories, Burlingame, $\mathrm{CA}$ ) for $1.5 \mathrm{~h}$ at room temperature, followed by reaction with avidin-biotin complex (ABC, Vector Elite kit; 1:200; Vector Laboratories). Sections were developed with Nickel-diaminobenzidine tetrahydrochloride (Ni-DAB), which produced a black reaction product in cell nuclei. There was no nuclear staining in the absence of primary antiserum. To stain for GAD, we used mouse anti-GAD67 monoclonal antibody (MAB5406, lot \# 25030191; Chemicon, Temecula, CA). This antibody (raised against recombinant GAD67 protein) is specific for GAD67 and shows no reactivity to GAD65 by Western blot (manufacturer's technical information). The antibody has been used successfully for Western blot and immunohistochemistry on rat and mouse tissue (manufacturer's technical information). Sections were incubated into the primary antibody $(1: 300)$ at $4^{\circ} \mathrm{C}$ over $48 \mathrm{~h}$, then processed with biotinylated anti-mouse IgG (BA-2001, 1:500; Vector Laboratories), followed by reaction with avidin-biotin complex (Avidin/biotin blocking kit, 1:100; Vector Laboratories) and developed with DAB to produce a brown reaction product. Omission of the GAD primary antiserum resulted in the absence of specific staining. We always performed matched immunological processing of tissue simultaneously on pairs of brains from the different experimental groups. After the staining, the sections

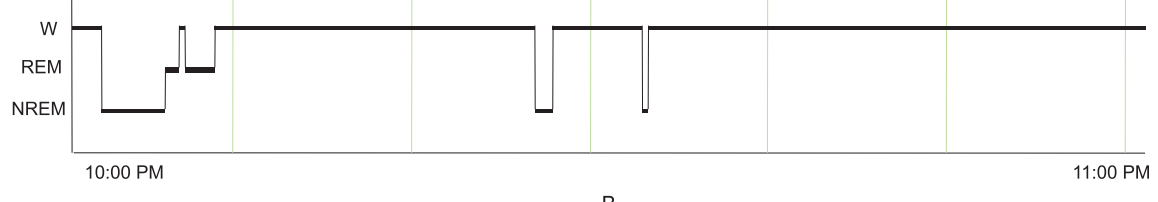

B

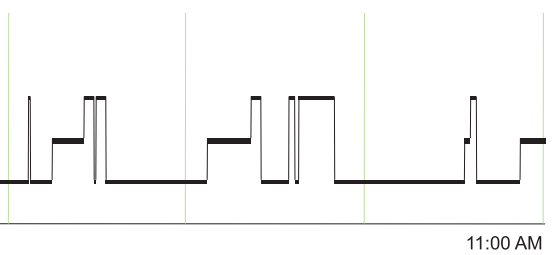

C $100 \mathrm{AM}$ $1: 00 \mathrm{AM}$ D and coverslipped with Depex.

\section{Data analysis}

Sleep analysis. Sleep-wakefulness states of the rats were determined by an experienced scorer on the basis of the predominate state within each $10 \mathrm{~s}$ epoch. The scorer was blind to experimental condition and group identity of the animal. Wakefulness was defined by the presence of lowamplitude and high-frequency EEG activity combined with elevated neck muscle tone. Non-REM sleep consisted of a high-amplitude slow-wave EEG together with a low-EMG tone relative to wakefulness. REM sleep was identified by the presence of a moderate-amplitude EEG with dominant theta-frequency activity coupled with minimal neck EMG tonus except for occasional brief twitches.

During sleep deprivation, sleep pressure was defined by counting the number of sleep entries within the experimental procedure. To track the accumulation of sleep pressure, the number of sleep entries in each animal was averaged per consecutive $10 \mathrm{~min}$ interval of the $2 \mathrm{~h}$ sleep deprivation period.

Cell counts. Cell counts were conducted by an individual who was blind to the experimental condition of the animals. The Neurolucida computer-aided plotting system (MicroBrightField, Williston, VT) was used to identify and quantify neurons that were single labeled for c-Fos-IR and double-labeled for Fos plus GAD-IR. Section outlines were drawn under $20 \times$ magnification. Fos IRNs and Fos plus GAD IRNs were mapped in the section outlines under $400 \times$ magnification. All cell counts were calculated for constant rectangular grids corresponding to four areas of interest. (1) The rostral MnPN (rMnPN) grid was a $600 \times 600$ $\mu \mathrm{m}$ square, centered on the apex of the third ventricle rostral to the decussation of the anterior commissure and to bregma (anterior, 0.1 
mm) (Gong et al., 2000). (2) The caudal MnPN (cMnPN) grid was placed immediately dorsal to the third ventricle at the level of the decussation of the anterior commissure, extending $150 \mu \mathrm{m}$ laterally and $600 \mu \mathrm{m}$ dorsally just caudal to bregma (anterior, $-0.26 \mathrm{~mm}$ ) (Gong et al., 2000). The vlPOA counting grid was placed at the level $160 \mu \mathrm{m}$ or more caudal to the organum vasculosum of the lamina terminalis (approximately anterior, from -0.3 to $-0.7 \mathrm{~mm}$ relative to bregma), and was subdivided into core and extended sections. (3) The vlPOA core box was $300 \mu \mathrm{m}$ wide by 300 $\mu \mathrm{m}$ high, placed along the base of the brain, with its far border $400 \mu \mathrm{m}$ lateral to the lateral edge of the optic chiasm. (4) The medial extended vlPOA box was medial to the vlPOA core, $400 \mu \mathrm{m}$ wide by $300 \mu \mathrm{m}$ high. The dorsal extended vlPOA box was $200 \mu \mathrm{m}$ wide by $300 \mu \mathrm{m}$ high, positioned above the vlPOA core and medial extended vlPOA boxes and centered over their border.

For both the rMnPN and the cMnPN, cell counts were made in three sections and averaged to yield a single value for each rat. For vlPOA, cell counts were made bilaterally in three sections containing the largest part of the vlPOA. Those six counts were then averaged to yield a single value for both the vlPOA core and extended vlPOA (medial and dorsal sections combined) for each rat.

Statistical analysis. All results are reported as mean \pm SEM. One-way nonrepeated-measures ANOVA was calculated for behavioral stage percentages across animals that were allowed spontaneous sleep-waking behavior during the light period and in the dark and the light sleepdeprived rats (Table 1). A similar ANOVA was calculated for the number of attempts to enter into sleep across all groups of sleep-deprived (light SLD, experiment 1; light SLD, experiment 2; and dark SLD, experiment 2) rats (Table 2).

For single Fos-IR cell counts in MnPN and vlPOA sites across all four groups of experiment 1, a one-way nonrepeated-measures ANOVA was calculated (Figs. 5A, 6A). A similar ANOVA was calculated for Fos plus GAD IRNs in MnPN and vlPOA sites across the same groups of rats (Figs. $5 B, 6 B)$ and for Fos-IR cell counts across all groups of sleep-deprived rats (See Table 2). After all ANOVAs, significance of the differences between individual group means was assessed by Newman-Keuls post hoc tests.

\section{Results}

Experimental rats $(n=33)$ showed different profiles of their sleep-waking diurnal organization estimated by percentage time spent in sleep during the light/rest period versus the dark/active period. A large group of rats $(n=24)$ exhibited strong diurnal rhythms in sleep amounts during $24 \mathrm{~h}$ baseline recordings; mean percentage of sleep time during the light period was $64.87 \pm$ $1.6 \%$, whereas the percentage of sleep time during the dark period was $26.99 \pm 3.0 \%$. These 24 rats were the subjects for experiment 1 . Nine rats did not exhibit diurnality in sleep-waking behavior during $24 \mathrm{~h}$ baseline recordings; the mean percentage of sleep time during the light period $(50.4 \pm 2.6)$ was not significantly different from the percentage of sleep time during the dark period $(47.35 \pm 1.9 \%)$. These rats were the subjects for experiment 2. Figure 1 presents mean sleep-waking amounts during $24 \mathrm{~h}$ baseline recordings for six rats exhibiting strong diurnal rhythms in sleep-waking $(A)$ and six rats exhibiting weak or absent diurnal rhythms in sleep and waking $(B)$.

\section{Experiment 1}

Rats $(n=6)$ that were allowed spontaneous sleep and waking during the dark period manifested low percentage of sleep ( $8.8 \pm$ $3.4 \%$ ) during $1 \mathrm{~h}$ before death whereas the light period recorded rats $(n=6)$ exhibited $73.7 \pm 2.6 \%$ sleep (Table 1, Fig. $2 A, B$ ). Sleep-deprived rats accumulated $5.6 \pm 1.4 \%$ of non-REM sleep and no REM sleep during the deprivation period (Table 1, Fig. $2 C)$. Rats $(n=6)$ that were allowed a $1 \mathrm{~h}$ of recovery sleep after sleep deprivation during the light period exhibited $88.9 \pm 1.3 \%$ sleep (Table 1, Fig. 2D).

All sleep-deprived rats $(n=12)$ manifested evidence of grad-
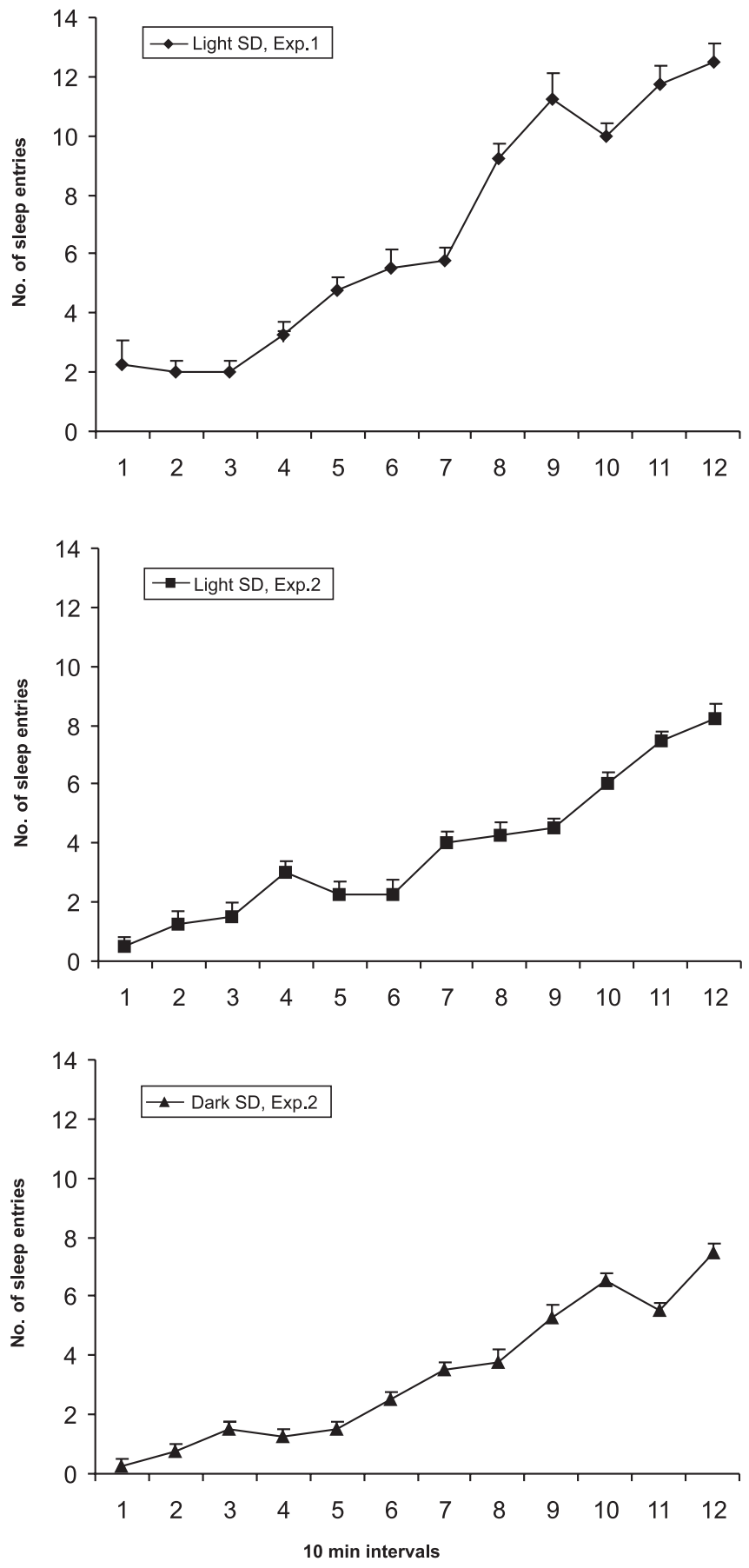

Figure 3. Average numbers of attempts to enter sleep during consecutive 10 min intervals across $2 \mathrm{~h}$ periods of total sleep deprivation for rats with different patterns of baseline sleepwaking diurnal organization. $A$, Sleep deprivation during the light period in the condition of a strong diurnal rhythm in baseline sleep and waking (light SLD, experiment $1 ; n=6$ ). $\boldsymbol{B}$, Sleep deprivation during the light period in the condition of a weak diurnal rhythm in baseline sleep and waking (light SLD, experiment 2; $n=5$ ). C, Sleep deprivation during the dark period in the condition of a weak diurnal rhythm in baseline sleep-waking (dark SLD, experiment $2 ; n=4$ )

ual elevation of sleep pressure; the number of sleep entries progressively increased within the deprivation period (Fig. 3). Both total sleep time and the number of sleep onsets were significantly elevated in $1 \mathrm{~h}$ recovery sleep animals compared with animals exhibiting spontaneous sleep in the light (Table 1). Rebound increases in these measures during recovery sleep provide further evidence that sleep pressure was elevated at the end of the $2 \mathrm{~h}$ sleep deprivation period. 
Examples of the distribution of Fosand Fos plus GAD-IR neurons in the $\mathrm{MnPN}$ and vlPOA for representative animals are shown in Figure 4. Mean numbers of single Fos-IR and Fos plus GAD double IR neurons in MnPN and vlPOA sites across all four groups of animals are shown in Figures 5 and 6 . The numbers of Fos plus GAD double-IR neurons in rostral and caudal MnPN were highest in sleepdeprived rats that were not allowed a recovery sleep and lowest in rats permitted sleep during the dark cycle (Fig. $5 B$ ). In vlPOA sites, Fos plus GAD double-IR cell counts were highest in rats permitted sleep during the light period and sleep-deprived rats that were allowed recovery sleep after the deprivation (Fig. 6B). See Figures 5 and 6 and the accompanying legends for a summary of other between group differences.

\section{Experiment 2}

Light-sleep deprived $(n=5)$ and darksleep deprived $(n=4)$ rats exhibited similar degrees of sleep homeostatic pressure estimated by total numbers of sleep entries within the experimental period (Table 2). As shown, the number of attempts to initiate sleep in these two groups of rats was significantly lower compared with the number of sleep attempts in rats that were sleep-deprived during the light/rest period in experiment 1 . However, sleepdeprived rats in experiment 2 manifested evidence of gradual accumulation of sleep pressure; the number of required awakenings progressively increased within the deprivation period (Fig. 3).

The numbers of single Fos-IR and Fos plus GAD double-IR neurons in the MnPN of light-sleep and dark-sleep deprived rats in experiment 2 were not significantly different, but values were lower than those for rats that were subjected to deprivation during the light period under the condition of a strong diurnal rhythm in baseline sleep and waking (Table 2).

\section{Discussion}

Neuronal substrates underlying homeostatic aspects of sleep regulation are poorly defined. We report for the first time that GABAergic neurons in the MnPN exhibit maximal expression of Fos-IR in situations of high homeostatic sleep need accompanied by minimal sleep (i.e., in response to sleep deprivation during the light/rest period) (Fig. 5B). In contrast, vlPOA GABAergic neurons exhibit maximal Fos-IR during recovery sleep and spontaneous sleep in the light (Fig. $6 \mathrm{~B}$ ). These results identify a potential mechanistic role for MnPN GABAergic neurons in homeostatic sleep regulation.

Across the several conditions studied in experiments 1 and 2, dissociation of sleep pressure, sleep amount, and time of day were achieved. In experiment 1, Fos-IR in MnPN GABAergic neurons was lowest during spontaneous sleep in the dark, a condition of low sleep pressure and low sleep amount. However, in a condition of high sleep pressure and minimal sleep (sleep deprivation in the light period), Fos-IR in MnPN GABAergic neurons was maximal. In two conditions of high sleep amount, spontaneous sleep and recovery sleep in the light, Fos-IR in MnPN GABAergic neurons was higher in the condition with higher sleep pressure (i.e., recovery sleep). Collectively, these results demonstrate that activation of MnPN GABAergic neurons varies along the dimension of increasing sleep pressure.

Fos-IR in vlPOA GABAergic neurons was significantly higher during both spontaneous sleep and recovery sleep, compared with sleep deprivation (Fig. 6). This agrees with previous reports that Fos-IR in vlPOA neurons does not increase after sleep deprivation unless animals are permitted recovery sleep (Sherin et al.1996), and that sleep-related discharge of vlPOA neurons is elevated after $16 \mathrm{~h}$ of sleep deprivation, whereas waking-related discharge rates are unchanged (Szymusiak et al., 1998). Fos-IR in vlPOA GABAergic neurons is significantly elevated during recovery sleep after $24 \mathrm{~h}$ sleep deprivation compared with baseline sleep (Gong et al., 2004), supporting unit recording data that sleep-related activation of the vlPOA can occur in response to increasing homeostatic sleep pressure. Several lines of evidence support the hypothesis that mutual inhibitory interactions between vlPOA sleep-regulatory neurons and monoaminergic systems function to stabilize sleep and waking states (Saper et al., 2001, 2005). Increased activation of vlPOA GABAergic neurons during recovery sleep after sustained sleep loss could function to prolong sleep bout durations and to promote deeper sleep stages via inhibition of monoaminergic systems.

Reduced Fos-IR in MnPN neurons during spontaneous sleep in the dark versus the light period is interpreted by us to reflect low sleep pressure during the dark period. Alternatively, low 
A

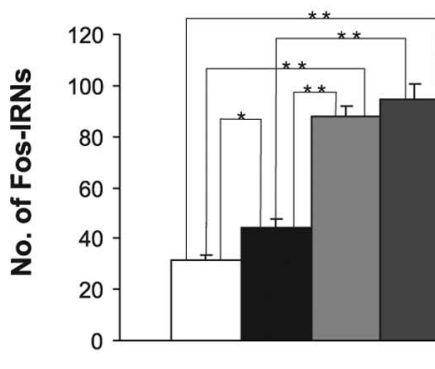

rMnPN

B

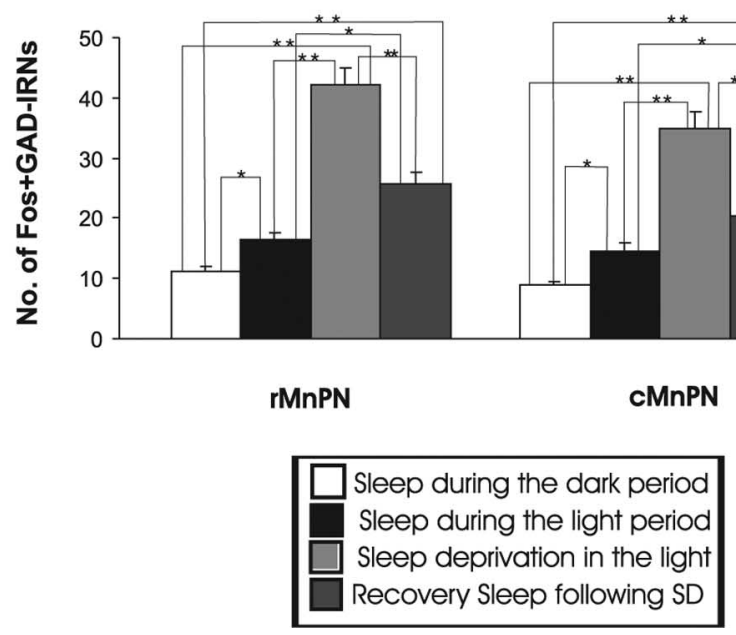

Figure 5. Mean numbers of Fos IRNs $(\boldsymbol{A})$ and Fos plus GAD IRNs $(\boldsymbol{B})$ in rMnPN and cMnPN, respectively, across all four groups of rats from experiment 1 . For single Fos-IR cell counts in the MnPN, ANOVA indicated significant effects of experimental conditions in both the rostral $\left(F_{(3,20)}=59.57 ; p<0.001\right)$ and caudal $\left(F_{(3,20)}=50.28 ; p<0.001\right)$ portions of the MnPN. There were significant effects of experimental conditions for double Fos plus GAD-IR cell counts in both rostral $\left(F_{(3,20)}=56.15 ; p<0.001\right)$ and caudal $\operatorname{MnPN}\left(F_{(3,20)}=41.37 ; p<0.001\right)$. Individual group mean differences as determined by post hoc tests (Newman-Keuls) are indicated by asterisks $\left({ }^{*} p<0.01\right.$; $\left.{ }^{* *} p<0.001\right)$.

Fos-IR cell counts in the dark could be a consequence of lightdark modulation of $c$-fos expression in this nucleus. However, results of experiment 2 do not support such an explanation. Rats with weak diurnal rhythms exhibited similar levels of sleep pressure, defined by the number of attempts to initiate sleep, during SLD in the light period and SLD in the dark period (Table 2). That Fos plus GAD-IR cell counts did not differ in these two conditions (Table 2) is evidence that in the absence of diurnal variation in sleep pressure, no strong light-dark rhythm of $c$-fos expression is evident in MnPN neurons.

We previously reported low levels of Fos-IR in the MnPN after $2 \mathrm{~h}$ of sleep deprivation in the light period (Gong et al., 2000), in contrast to this study and a recent publication (Gvilia et al., 2005). We can identify two important differences in the methods of sleep deprivation among these studies. In our recent studies, animals were adapted to sleep deprivation procedures during the 7-9 d before experiments, and stimuli were applied at the minimal intensity required to produce arousal. In our original report (Gong et al., 2000), animals were not adapted to deprivation procedures, and no attempts were made to keep arousing stimuli at threshold levels. Therefore, it may be that hyperarousal induced by novel, intense stimuli during sleep deprivation evokes strong inhibition of MnPN sleep-active neurons, such that the

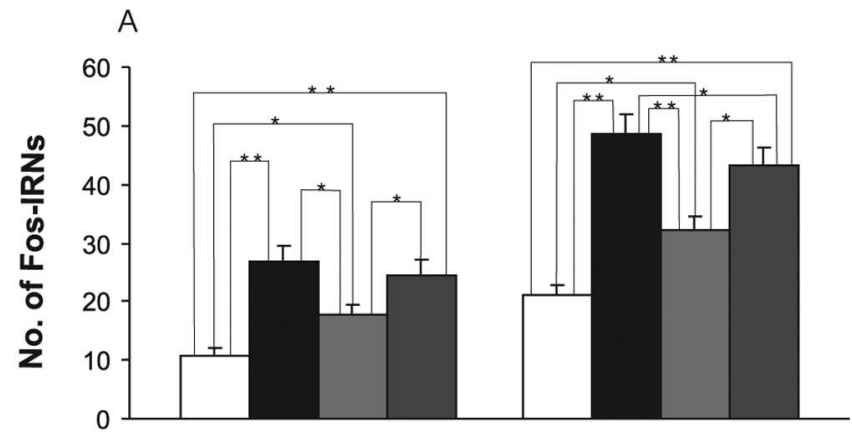

vIPOA cluster

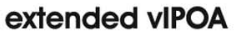

B
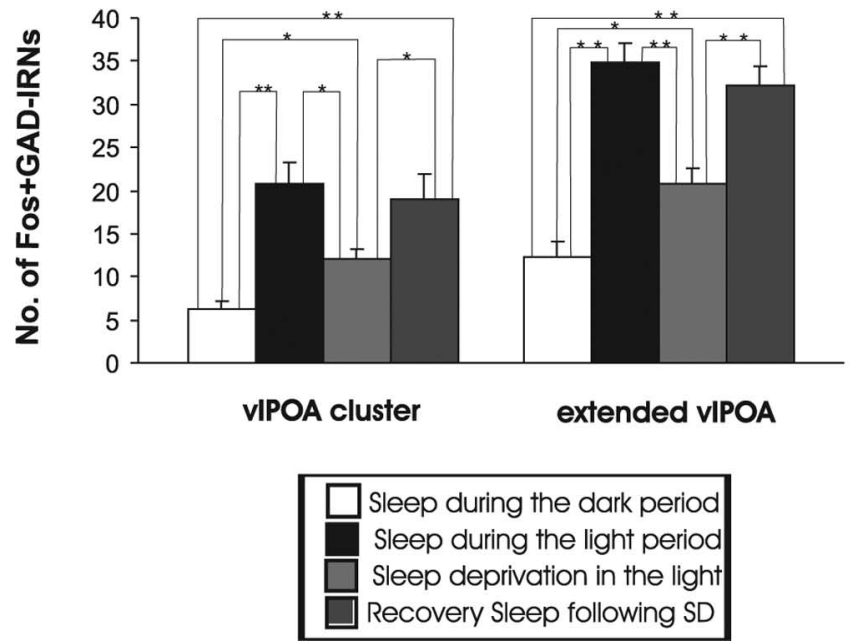

Figure 6. Mean numbers of Fos IRNs $(\boldsymbol{A})$ and Fos plus GAD IRNs $(\boldsymbol{B})$ in vIPOA cluster and extended vIPOA, respectively, across all four groups of experiment 1 . For single Fos-IR cell counts in the vIPOA, ANOVA indicated significant effects of experimental conditions in both the vIPOA cluster $\left(F_{(3,20)}=11.37 ; p<0.001\right)$ and extended vIPOA $\left(F_{(3,20)}=22.35 ; p<0.001\right)$. There were significant effects of experimental conditions for double Fos plus GAD-IR cell counts in both the vIPOA cluster $\left(F_{(3,20)}=11.51 ; p<0.001\right)$ and extended vIPOA $\left(F_{(3,20)}=26.99 ; p<\right.$ 0.001 ). Individual group mean differences as determined by post hoctests (Newman-Keuls) are indicated by asterisks $\left({ }^{*} p<0.01\right.$; $\left.{ }^{* *} p<0.001\right)$.

excitatory effects of increasing homeostatic pressure cannot be detected by changes in Fos-IR.

It has been reported that more MnPN GABAergic neurons express Fos-IR after $3 \mathrm{~h}$ of recovery sleep compared with $3 \mathrm{~h}$ of sleep deprivation (Modirrousta et al., 2004). The finding that few MnPN GABAergic neurons express Fos-IR after sleep deprivation appears contradictory to our current findings. At least two differences in methodology could account for the different results. First, the timing of sleep deprivation differed. We performed sleep deprivation beginning at 9:00 A.M., $1 \mathrm{~h}$ after lightson. Modirrousta et al. (2004) deprived animals of sleep beginning at 12:00 P.M. The time of lights-on was not specified in this study, but sleep deprivation may have been applied after the major sleep period of the light cycle, when overall sleep pressure was low. Second, Modirrousta et al. (2004) report that no sleep occurred during their sleep deprivation procedures, suggesting that animals were continually handled during deprivation. We applied arousing stimuli only after animals exhibited brief, EEG-defined sleep onsets. Increased Fos-IR in MnPN GABAergic neurons in our study may have reflected neuronal discharge during wake to sleep transitions (Suntsova et al., 2002). 
We reported previously that neurons in the MnPN and vlPOA express c-Fos-IR under conditions of increasing REM sleep homeostatic pressure (Gvilia et al., 2006). Significant reductions in REM sleep time were achieved during a $2 \mathrm{~h}$ period of imposed REM sleep restriction, a procedure that did not result in changes in non-REM sleep time. c-Fos activation in the MnPN was maximal in rats exhibiting the highest number of attempts to initiate REM sleep during the REM restriction period. Fos-IR was also elevated during recovery sleep after REM sleep restriction, compared with baseline sleep. In the MnPN, increased REM sleep pressure was associated with increased Fos-IR in non-GABAergic neurons (Gvilia et al., 2006), in contrast to the activation of predominantly GABAergic neurons reported here as a consequence of total sleep deprivation (Fig. 5).

Studies of sleep-related neuronal discharge suggest different functional roles for vlPOA and MnPN neurons in sleep regulation, consistent with the results of Fos-IR studies reported here. Most sleep-active vlPOA neurons display increased activity during the immediate transition from waking to sleep and become progressively activated from light to deep non-REM sleep (Szymusiak et al., 1998). Sleep-related discharge rates of vlPOA neurons are elevated in rats after $16 \mathrm{~h}$ of sleep deprivation compared with nondeprived rats, but waking discharge rates are unchanged (Szymusiak et al., 1998). Most sleep-active MnPN neurons show gradual increases in firing rate well in anticipation of sleep onset (Suntsova et al., 2002). Peak discharge rates of MnPN neurons are observed early in the development of non-REM sleep episodes and rates decline across sustained sleep episodes in the absence of intervening waking (Suntsova et al., 2002). Collectively, findings suggest that GABAergic neurons in the MnPN become progressively activated in response to increasing homeostatic sleep pressure accruing during sustained wakefulness and function to promote transitions from waking to sleep. Sleep-related activation of GABAergic neurons in the vlPOA may function primarily to consolidate sleep and to regulate sleep depth within a sleep episode.

Anatomical and physiological evidence suggests that MnPN and vlPOA neurons function to promote sleep via descending inhibitory modulation of arousal systems located in the posterior hypothalamus and brainstem. The vlPOA heavily innervates wake-promoting histaminergic neurons in the tuberomammillary nucleus (TMN) (Sherin et al., 1996, 1998). Electrical stimulation of the vIPOA area in a horizontal rat brain slice preparation evokes GABA-mediated inhibitory postsynaptic potentials in histaminergic neurons in the TMN (Yang and Hatton, 1997). The vlPOA projects to the locus ceruleus and dorsal raphe nucleus (Sherin et al., 1998; Steininger et al., 2001), and to the ventral periaqueductal gray, an area that contains wake-promoting dopaminergic neurons (Lu et al., 2006). Projections from the vlPOA and the MnPN to hypocretin neuronal field in the perifornical region of the lateral hypothalamus (PFLH) have been documented (Yoshida et al., 2006). Projection neurons from both the MnPN and the vlPOA to the PFLH express c-Fos protein-IR during sleep (Uschakov et al., 2006). Electrical stimulation of the MnPN evokes suppression of waking discharge in PFLH neurons (Suntsova et al., 2003) and inhibition of preoptic area neurons by local infusion of muscimol induces Fos-IR in hypocretin neurons (Satoh et al., 2003). We hypothesize that elevation of homeostatic sleep pressure occurring as a consequence of sustained waking leads to enhanced GABA-mediated inhibition of monoaminergic, hypocretinergic, and dopaminergic arousal systems via activation of MnPN and vlPOA neurons.

The factor(s) responsible for activation of $\mathrm{MnPN}$ neurons during sleep deprivation and of vlPOA neurons during recovery sleep are unknown. The endogenous somnogen adenosine has been implicated in homeostatic sleep regulation and may play a role in regulating changes in vlPOA and $\mathrm{MnPN}$ neuronal activity in response to sustained wakefulness. Adenosine disinhibits vlPOA neurons in vitro via $\mathrm{A}_{1}$ adenosine receptors (Chamberlin et al., 2003) and directly excites vlPOA neurons in vitro via $A_{2 A}$ receptors (Gallopin et al., 2005). Infusion of $\mathrm{A}_{2 \mathrm{~A}}$ receptor agonists adjacent to the vlPOA (Methippara et al., 2005) and into the ventral striatum immediately rostral to the MnPN (Satoh et al., 2006) promote sleep. The cytokine interleukin- $1 \beta$ (IL-1 $\beta$ ) has also been implicated as a homeostatic sleep factor (Obal and Krueger, 2003), and ICV administration of IL-1 $\beta$ promotes sleep and activates Fos-IR in MnPN neurons (Baker et al., 2005).

\section{References}

Baker FC, Shah S, Stewart D, Angara C, Gong H, Szymusiak R, Opp MR, McGinty D (2005) Interleukin-1-beta enhances non-rapid eye movement sleep and increases c-Fos protein expression in the median preoptic nucleus of the hypothalamus. Am J Physiol 288:R998-R1005.

Chamberlin NL, Arrigoni E, Chou TC, Scammell TE, Green RW, Saper CB (2003) Effects of adenosine on GABAergic synaptic inputs to identified ventrolateral preoptic neurons. Neuroscience 119:913-918.

Dragunow M, Faull R (1989) The use of c-fos as a metabolic marker in neuronal pathway tracing. J Neurosci Methods 29:261-265.

Gallopin T, Luppi PH, Cauli B, Urade Y, Rossier J, Hayaishi O, Lambolez B, Fort P (2005) The endogenous somnogen adenosine excites a subset of sleep-promoting neurons via A2A receptors in the ventrolateral preoptic nucleus. Neuroscience 134:1377-1390.

Gaus SE, Strecker RE, Tate BA, Parker RA, Saper CB (2002) Ventrolateral preoptic nucleus contains sleep-active, galaninergic neurons in multiple mammalian species. Neuroscience 115:285-294.

Gong H, Szymusiak R, King J, Steininger T, McGinty D (2000) Sleep-related c-Fos protein expression in the preoptic hypothalamus: effects of ambient warming. Am J Physiol Regul Integr Comp Physiol 279:R2079-R2088.

Gong H, McGinty D, Guzman-Marin R, Chew KT, Stewart D, Szymusiak R (2004) Activation of c-fos in GABAergic neurons in the preoptic area during sleep and in response to sleep deprivation. J Physiol (Lond) 556:935-946.

Gvilia I, Angara C, McGinty D, Szymusiak R (2005) Different neuronal populations of the rat median preoptic nucleus express $c$-fos during sleep and in response to hypertonic saline or angiotensin-II. J Physiol (Lond) 569:587-599.

Gvilia I, Turner A, McGinty D, Szymusiak R (2006) Preoptic area neurons and the homeostatic regulation of rapid eye movement sleep. J Neurosci 26:3037-3044.

Lu J, Jhou TC, Saper CB (2006) Identification of wake-active dopaminergic neurons in the ventral periaqueductal gray matter. J Neurosci 26:193-202.

Methippara MM, Kumar S, Alam MN, Szymusiak R, McGinty D (2005) Effects on sleep of microdialysis of adenosine A1 and A2A receptor analogs into the lateral preoptic area of rats. Am J Physiol 289:R1715-R1723.

Modirrousta M, Mainville L, Jones B (2004) Gabaergic neurons with alpha2-adrenergic receptors in basal forebrain and preoptic area express c-Fos during sleep. Neuroscience 129:803-810.

Morgan JI, Curran T (1986) Role of ion flux in the control of c-fos expression. Nature 322:552-555.

Obal Jr F, Krueger JM (2003) Biochemical regulation of non-rapid-eyemovement sleep. Front Biosci 1:d520-d550.

Peterfi Z, Churchill L, Hajdu I, Obal Jr F, Krueger JM, Parducz A (2004) Fos-immunoreactivity in the hypothalamus: dependency on the diurnal rhythm, sleep, gender, and estrogen. Neuroscience 124:695-707.

Saper CB, Chou TC, Scammell TE (2001) The sleep switch: hypothalamic control of sleep and wakefulness. Trends Neurosci 24:726-731.

Saper CB, Cano G, Scammell TE (2005) Homeostatic, circadian and emotional regulation of sleep. J Comp Neurol 493:92-98.

Satoh S, Matsumura H, Nakajima T, Nakahama K, Kanbayashi T, Nishino S, Yoneda H, Shigeyoshi Y (2003) Inhibition of rostral basal forebrain neurons promotes wakefulness and induces FOS in orexin neurons. Eur J Neurosci 17:1635-1645. 
Satoh S, Matsumura H, Kanbayashi T, Yoshida Y, Urakami T, Nakajima T, Kimura N, Nishino S, Yoneda H (2006) Expression of FOS in orexin neurons during sleep induced by adenosine $\mathrm{A}(2 \mathrm{~A})$ agonist. Behav Brain Res 170:277-286.

Sherin JE, Shiromani PJ, McCarley RW, Saper CB (1996) Activation of ventrolateral preoptic neurons during sleep. Science 271:216-219.

Sherin JE, Elmquist JK, Torrealba F, Saper CB (1998) Innervation of histaminergic tuberomammillary neurons by GABAergic and galaninergic neurons in the ventrolateral preoptic nucleus of the rat. J Neurosci 18:4705-4721.

Steininger T, Gong H, McGinty D, Szymusiak R (2001) Subregional organization of preoptic area/anterior hypothalamic projections to arousalrelated monoaminergic cell groups. J Comp Neurol 429:638-653.

Suntsova N, Szymusiak R, Alam MN, Guzman-Marin R, McGinty D (2002) Sleep-waking discharge patterns of median preoptic nucleus neurons in rats. J Physiol (Lond) 543:665-667.
Suntsova N, Guzman-Marin R, Alam MN, Szymusiak R, Shouse M, McGinty D (2003) EEG, behavioral and neuronal effects of median preoptic nucleus electrical stimulation. Sleep 26:A47.

Szymusiak R, Alam MN, Steininger TL, McGinty D (1998) Sleep-waking discharge patterns of ventrolateral preoptic/anterior hypothalamic neurons in rats. Brain Res 803:178-188.

Uschakov A, Gong H, McGinty D, Szymusiak R (2006) Sleep-active neurons in the preoptic area project to the hypothalamic paraventricular nucleus and the perifornical lateral hypothalamus. Eur J Neurosci 23: 3284-3296.

Yang Q, Hatton G (1997) Electrophysiology of excitatory and inhibitory afferents to rat histaminergic tuberomammillary nucleus neurons from hypothalamic and forebrain sites. Brain Res 773:162-172.

Yoshida K, McCormack S, Espana R, Crocker A, Scammell T (2006) Afferents to the orexin neurons of the rat brain. J Comp Neurol 494: 845-861. 